\title{
PETERSON-STEIN FORMULAS IN THE ADAMS SPECTRAL SEQUENCE
}

\author{
WARREN M. KRUEGER
}

Abstract. The purpose of this note is to establish Peterson-Stein formulas for second order differentials in the Adams spectral sequence.

In [4], Peterson and Stein related certain values of secondary cohomology operations to the values of certain functional primary operations. Their method was that of universal example. Here using a different approach, we establish analogous formulas for second order and functional first order differentials which occur in the version of the Adams spectral sequence that is formulated with respect to $E_{*}$ homology in the stable homotopy category [1, p. 238]. (For these formulas we do not need the assumptions which serve to identify $E_{2}$ or assure convergence.)

We begin by recalling the definition of the Adams differential. In the spectral sequence for a pair of spectra $(X, Y)$ the group $E_{r}^{s, t}$ is a subquotient of $\left[X, E \wedge(C(i))^{s} \wedge Y\right]_{t}$ and $d_{r}^{s, t}: E_{r}^{s, t} \rightarrow E_{r}^{s+r, t+r-1}$ is induced by the additive relation

$$
\left(i_{s+r}\right)_{*}\left[\left(p_{s+r-1}\right)_{*} \circ \cdots \circ\left(p_{s+1}\right)_{*}\right]^{-1}\left(j_{s}\right)_{*} .
$$

Here $C(i)$ denotes the mapping cone of $i: S^{0} \rightarrow E, W^{s}$ denotes the $s$-fold smash product of $W$ with itself, and

$$
\begin{aligned}
& i_{k}:(C(i))^{k} \wedge Y \rightarrow E \wedge(C(i))^{k} \wedge Y, \\
& j_{k}: E \wedge(C(i))^{k} \wedge Y \rightarrow(C(i))^{k+1} \wedge Y
\end{aligned}
$$

and $p_{k}:(C(i))^{k+1} \wedge Y \rightarrow(C(i))^{k} \wedge Y$ are induced by smashing with the appropriate maps from $S^{0} \rightarrow E \rightarrow C(i) \rightarrow S^{0}$.

Now let $f: Y \rightarrow Y^{\prime}$ be a map in our category. Then we have

Proposition (P-S1). Let $u \in[X, E \wedge Y]_{0}$ belong to $\operatorname{ker} d_{1} \cap \operatorname{ker} f_{*}$. Then

$$
f_{*} d_{2}^{0}(u)=d_{1}^{1}\left(\left(d_{1}^{0}\right)_{f}(u)\right)
$$

in $\left[X, E \wedge(C(i))^{2} \wedge Y^{\prime}\right]_{1} / f_{*}\left(\operatorname{im} d_{1}^{1}\right)$.

Dually we have

Received by the editors May 1, 1975.

AMS (MOS) subject classifications (1970). Primary 55H 15.

Key words and phrases. Adams spectral sequence, differential, functional differential. 
Proposition (P-S2). Let $u \in[X, E \wedge Y]_{0}$ belong to $\operatorname{ker}\left(d_{1}^{0} f_{*}\right)$. Then

$$
\left(d_{1}^{1}\right)_{f}\left(d_{1}^{0}(u)\right)=d_{2}^{0}\left(f_{*}(u)\right)
$$

in $\left[X, E \wedge(C(i))^{2} \wedge Y^{\prime}\right]_{1} /\left(\operatorname{im} d_{1}^{1}+\operatorname{im} f_{*}\right)$.

The functional differentials here are formed the usual way via the Puppe sequence.

We give a proof of P-S2, the proof of P-S1 being dual in a certain sense. The argument depends on the "simultaneous solution" to two coextension problems which is given in the

LemMA. Let $X \stackrel{\rightarrow}{\longrightarrow} A \wedge B \stackrel{f \wedge g}{\longrightarrow} C \wedge D$ be a null-homotopic composition of maps of spectra. Then there are maps

$$
z_{1}: X \rightarrow C\left(1_{C} \wedge g\right) \text { and } z_{2}: X \rightarrow C\left(f \wedge 1_{D}\right)
$$

of degree +1 so that

(a) $Q\left(1_{C} \wedge g\right) z_{1} \simeq\left(f \wedge 1_{B}\right) z$ and $Q\left(f \wedge 1_{D}\right) z_{2} \simeq\left(1_{A} \wedge g\right) z$

(b) the compositions

$$
\begin{aligned}
& X \stackrel{z_{1}}{\longrightarrow} C\left(1_{C} \wedge g\right) \rightarrow C(f) \wedge C(g) \\
& X \stackrel{z_{2}}{\longrightarrow} C\left(f \wedge 1_{D}\right) \rightarrow C(f) \wedge C(g)
\end{aligned}
$$

are homotopic.

In the above and what follows we adopt the following notation for the Puppe sequence:

$$
W \stackrel{h}{\longrightarrow} Z \stackrel{P(h)}{\longrightarrow} C(h) \stackrel{Q(h)}{\longrightarrow} W .
$$

(We also note that this lemma corrects Proposition 5 of [3] from which Propositions 1 and 2 now follow except the minus signs in their statements disappear.)

Proof of Lemma. There are coextensions $z_{1}, z_{2}$ and $z^{\prime}$ of $\left(f \wedge 1_{B}\right) z$, $\left(1_{A} \wedge g\right) z$ and $z$ which are formed in the obvious way so that the following diagram

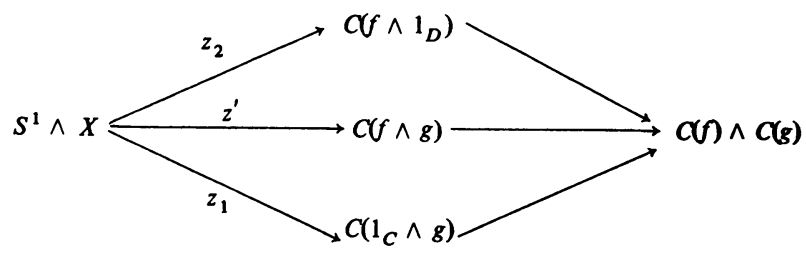

is homotopy commutative. (The unmarked arrows designate the obvious maps.)

Proof OF P-S2. For convenience we display the maps defining the differentials occurring in the formula. 


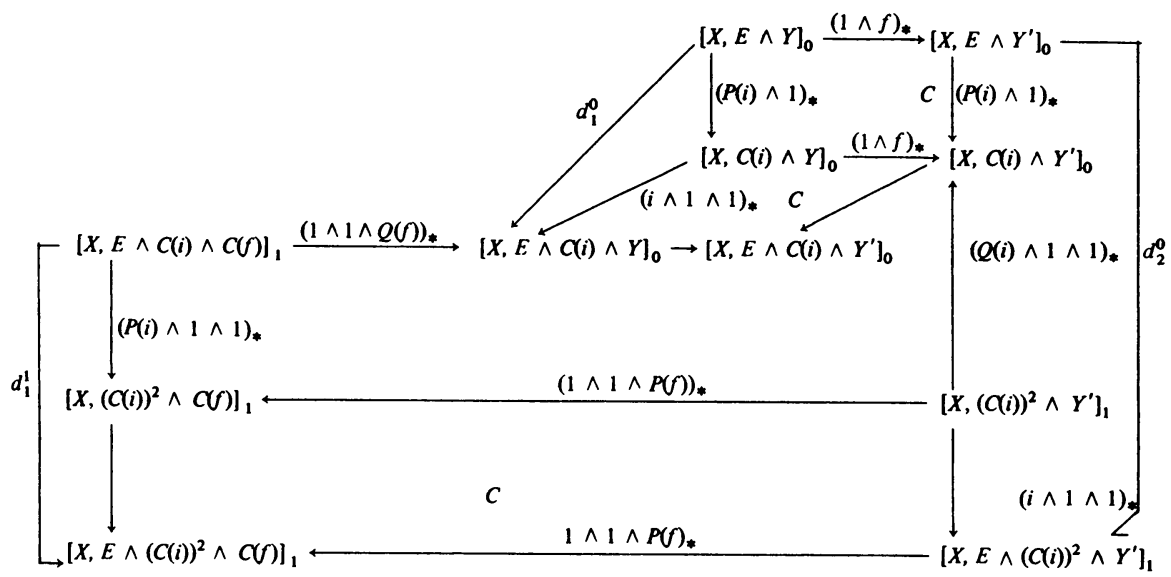

In this diagram the quadrilaterals marked with a " $C$ " are commutative.

Now apply the lemma to $z=(P(i) \wedge 1)_{*}(u)$ and $f \wedge g=i \wedge(1 \wedge f)$ : $S^{0} \wedge(C(i) \wedge Y) \rightarrow E \wedge\left(C(i) \wedge Y^{\prime}\right)$. Then there are elements

$$
z_{1} \in[X, E \wedge C(i) \wedge C(f)]_{1} \text { and } z_{2} \in\left[X,(C(i))^{2} \wedge Y^{\prime}\right]_{1}
$$

so that

$$
(1 \wedge 1 \wedge Q(f))_{*}\left(z_{1}\right)=d_{1}^{0}(u)
$$

and

$$
(Q(i) \wedge 1 \wedge 1)_{*}\left(z_{2}\right)=(P(i) \wedge 1)_{*}(1 \wedge f)_{*}(u)
$$

and

$$
(P(i) \wedge 1 \wedge 1)_{*}\left(z_{1}\right)=(1 \wedge 1 \wedge P(f))_{*}\left(z_{2}\right) .
$$

Then $(i \wedge 1 \wedge 1)_{*}\left(z_{2}\right)$ represents both $d_{2}^{0}\left(f_{*}(u)\right)$ and $\left(d_{1}^{1}\right)_{f}\left(d_{1}^{0}(u)\right)$ according to their definitions.

The indeterminacy in the formula is simply the larger of the indeterminacies of the two operations.

In a future paper involving Browder's work on the Kervaire invariant problem [2], we make strong use of P-S2.

\section{REFERENCES}

1. J. F. Adams, Stable homotopy and generalized homology, Mathematical Lecture Notes, University of Chicago, Chicago, 1971.

2. W. Browder, The Kervaire invariant of framed manifolds and its generalization, Ann. of Math. (2) 90 (1969), 157-186. MR 40 \#4963.

3. W. Krueger, Generalized Steenrod-Hopf invariants for stable homotopy theory, Proc. Amer. Math. Soc. 39 (1973), $609-615$.

4. F. P. Peterson and N. Stein, Secondary cohomology operations: two formulas, Amer. J. Math. 81 (1959), 281-305. MR 23 \# A1366.

Department of Mathematics, New Mexico State University, Las Cruces, New Mexico 88003 